\title{
THE CASSON-GORDON INVARIANTS IN HIGH-DIMENSIONAL KNOT THEORY
}

\author{
DANIEL RUBERMAN
}

\begin{abstract}
The Casson-Gordon invariants of knots in all dimensions are interpreted in terms of surgery theory. Applications are given to finding nondoubly slice knots, and doubly slice knots which are not the double of a disk knot. In even dimensions, the property of being doubly slice is shown to be largely homotopy theoretic, while in odd dimensions the surgery-theoretic method shows such properties to depend on more than the homotopy type.
\end{abstract}

In 1975, A. Casson and C. Gordon used the equivariant signatures defined in [AS] to show that classical knot cobordism differs radically from the high-dimensional theory. The author's thesis [R1] developed a generalization of the Casson-Gordon invariants to all odd dimensions to attack the problem of double null-concordance. (See also [St, Su, GL].) This is the question of whether an $n$-dimensional knot is a slice of an $(n+1)$-dimensional unknot. We showed further that analogous invariants could be defined in even dimensions and applied to the same problem.

In this paper, we give some new applications of the Casson-Gordon invariants in high-dimensional knot theory, and strengthen the results of [R1 and S1]. It was shown by J. Levine $[\mathbf{L}]$ that doubly slice knots are obtained if one doubles a disk pair along its boundary. Doubly slice knots have many properties in common with such doubled disk knots-in fact, according to [L], the concepts are equivalent for simple knots. Levine asked whether in fact all doubly slice knots were obtained as doubled disk knots and provided examples of knots in $S^{3}$ and $S^{4}$ which are not. In his Ph.D. thesis [S1] and more recent work [S2], L. Smolinsky gave examples in (higher) odd dimensions of knots which are doubly slice without being the double of a disk pair, using the Casson-Gordon invariants in a manner similar to [R1].

As an application of the even-dimensional invariants, we show that in all even dimensions there are doubly slice knots which are not doubled disk knots. To construct such examples, we show that an even-dimensional knot which has the $\mathbf{Z}[\mathbf{Z}]$-homology type of a doubly slice knot is also doubly slice. This contradicts a theorem of [R1] which asserts a bound on the even-dimensional Casson-Gordon invariants of a doubly slice knot. To reestablish the main result of [R1], namely that in all even dimensions there are "algebraically" doubly slice knots which are not doubly slice, we extend the definition of the even-dimensional invariants to a more general context than that used in [R1]. In that paper, $\mathbf{Q}$-valued invariants were defined for homomorphisms to a cyclic group $\mathbf{Z}_{d} ; d$ was assumed to be a prime power and only certain homomorphisms were allowed. In the approach taken in

Received by the editors December 12, 1986.

1980 Mathematics Subject Classification (1985 Revision). Primary 57Q45, 57M25, 57R67.

Key words and phrases. Doubly slice knots, surgery groups, $\alpha$-invariants.

Author partially supported by MSRI and NSF grant DMS 8502317 . 
this paper, composite $d$ (and arbitrary homomorphisms) are allowed-this yields $\mathbf{Q} / \mathbf{Z}$-valued invariants which are then shown to vanish for doubly slice knots.

In odd dimensions, we recast the Casson-Gordon invariants in terms of surgery theory, and interpret them as homomorphisms from certain $L$-groups. As a consequence of the realization of surgery obstructions, we find that there are knots whose complements are simple homotopy equivalent (relative to their boundaries) and such that one is doubly slice yet the other cannot be. This is in contrast to the behavior of even-dimensional knots (as remarked above) and also differs from ordinary knot concordance, where the homotopy type (rel boundary) determines the concordance class. Similarly, the property of being a doubled disk knot is shown not to be a homotopy property - this occurs in both even and odd dimensions.

ACKNOWLEDGEMENT. It is a pleasure to thank Larry Smolinsky and Jerry Levine for many helpful and interesting conversations about the material in this paper.

1. Casson-Gordon invariants. Using the $G$-signature theorem, Casson and Gordon [CG1, CG2] showed how to define an invariant of knots which admit a metabelian cover. In this section, we show how to interpret the Casson-Gordon invariant in many cases as a homomorphism from a certain surgery group. The advantage of this viewpoint is that it enables us to construct knots with nontrivial invariants by using the realization $[\mathbf{W}]$ of surgery groups and thereby to refine previous results concerning doubly slice knots and doubled disk knots. (Showing that the realization in the classical case actually produces a knot in $S^{3}$ requires some additional arguments.)

Notation. For $K$ a knot in $S^{n}, X($ or $X(K))$ will denote its exterior, and $Y$ will denote surgery on $K$, where we use the 0 -framed surgery if $n=3$. An infinite cyclic cover of either $X$ or $Y$ will be denoted with a subscript of " $\infty$ ", and $M_{k}$ will denote a $k$-fold branched cyclic cover. We will be discussing various surgery groups $L_{*}(G)$ and $\Gamma_{*}[\mathbf{Z}[G] \rightarrow \mathbf{Z}]$. Technically, we should label these $L_{*}^{s}$ or $L_{*}^{h}$, but the signature-type invariants we use in this paper are largely independent of the choice of $K$-theory. Thus we will be sloppy and just use the notation $L_{*}(G)$.

The original definition by Casson and Gordon of their invariant (extended to the higher dimensional case in [R1]) was essentially the following: Let $K$ be a knot in $S^{2 n-1}$, and $M_{k}$ its $k$-fold branched cover. If $f: H_{1}\left(M_{k}\right) \rightarrow \mathbf{Z}_{d}$ is a homomorphism, then $\sigma(K, f)$ was defined as the Atiyah-Singer alpha-invariant [AS] of the $\mathbf{Z}_{d}$-action on the induced cover of $M_{k}$. If $k=2$, then such a homomorphism corresponds to a dihedral representation of the knot group $\pi(K)=\pi_{1}(X(K))$. We give a surgery-group interpretation of the Casson-Gordon invariant in the case that the homomorphism $f$ comes from a representation onto a more general type of extension. The groups $G$ which we will use can be given by an extension

$$
1 \rightarrow \mathbf{Z}_{d} \rightarrow G \rightarrow \mathbf{Z} \rightarrow 1
$$

where $\mathbf{Z}$ acts by $t(g)=g^{a}$. Suppose $t$ has order $k$; then we get a group $G_{k}$ and a diagram

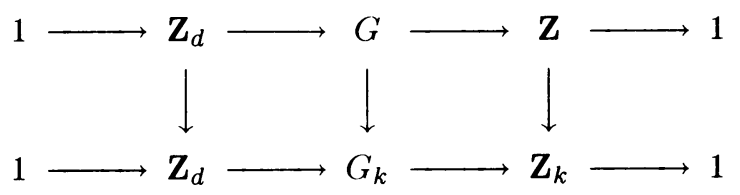


where the vertical maps are the natural surjections. If a knot group surjects to $G$, then there is a surjection $\varphi$ from $H_{1}\left(M_{k}\right)$ to $\mathbf{Z}_{d}$. In this paper we will consider only $\varphi$ 's which arise in this way. Note that in the special case that $1-a$ is invertible (as an integer mod $d$ ), then $G$ is in fact a high-dimensional knot group. This follows by computing $H_{1}(G)$ and $H_{2}(G)$ and using Kervaire's characterization $[\mathbf{K}]$ of such groups.

Consider the induced maps of surgery groups:

$$
L_{2 n}(G) \rightarrow L_{2 n}\left(G_{k}\right) \rightarrow L_{2 n}\left(\mathbf{Z}_{d}\right),
$$

where the last map is induced by the transfer. In geometric terms an element of $L_{2 n}(G)$ is represented by a normal map from a manifold to a Poincaré complex with $\pi_{1}=G$. Its image in $L_{2 n}\left(G_{k}\right)$ is given by the normal map induced by doing surgery to make the fundamental group of the Poincaré complex equal to $G_{k}$. The transfer of this latter element is then represented by the normal map between the $k$-fold covers induced by the surjection $G_{k} \rightarrow \mathbf{Z}_{k}$. In other words, the transfer of this element is the $\mathbf{Z}\left[\mathbf{Z}_{d}\right]$-valued intersection form of the induced $k$-fold covers.

We will denote by $R$ (or $R\left(\mathbf{Z}_{d}\right)$ as necessary) the $\operatorname{ring} \mathbf{Z}[\chi] / \Sigma$ where $\Sigma$ is the sum of the elements in the group $\mathbf{Z}_{d}$. Similarly, we will use the notation $\mathbf{Q} R$ (or $\left.\mathbf{Q} R\left(\mathbf{Z}_{d}\right)\right)$ for $R$ tensored with the rationals. These may be viewed as the ring of characters on $\mathbf{Z}_{d}$; the $\alpha$-invariant lies in the ring $\mathbf{Q} R$. Now the multisignature defines a map $[\mathbf{P}, \mathbf{W}] \rho: L_{2 n}\left(\mathbf{Z}_{d}\right) \rightarrow R\left(\mathbf{Z}_{d}\right)$. Denote the composite map from $L_{2 n}(G)$ to $R\left(\mathbf{Z}_{d}\right)$ by $C G$.

Suppose now that $K$ is a knot whose group $\pi(K)$ has a representation onto such an extension $G$, inducing a surjection $f: H_{1}\left(M_{k}\right) \rightarrow \mathbf{Z}_{d}$. If $x$ is an element of $L_{2 n}(G)$, then $x$ can be represented by a matrix with entries in $\mathbf{Z}[G]$, which can be thus lifted to a matrix with entries in the group ring of the knot group. (This matrix will in general not be invertible in $\mathbf{Z}[\pi]$, but this is not important.) If $n>2$, Wall's procedure [W] for realizing this element gives a cobordism of $X$ (rel boundary) to another manifold $X^{\prime}$, which will have $\pi_{1}\left(X^{\prime}\right)=\pi_{1}(X)$ and $H_{*}\left(X^{\prime}\right)=H_{*}(X)$ and so will also be the complement of a knot $K^{\prime}$ in $S^{2 n-1}$. It turns out that this works in the classical dimension $n=2$ as well, but this requires some care in the construction and is shown below in Theorem 1.2. By construction $\pi\left(K^{\prime}\right)$ will also have a surjection to $G$ which extends across the cobordism between $X$ and $X^{\prime}$.

THEOREM 1.1. The difference in Casson-Gordon invariants, $\sigma(K, f)$ $\sigma\left(K^{\prime}, f^{\prime}\right)=C G(x)$.

PROOF. The construction outlined above and in Theorem 1.2 provides a cobor$\operatorname{dism} W$ between the knot exteriors $X$ and $X^{\prime}$ which is a product near the boundary. The $k$-fold cyclic cover of $W, W_{k}$, can be completed to a compact manifold $\widehat{W}_{k}$ with boundary equal to $M_{k} \cup M_{k}^{\prime}$. Note that $\widehat{W}_{k}$ is a cobordism over $\mathbf{Z}_{d}$ and can thus be used to compute $\sigma(K, f)-\sigma\left(K^{\prime}, f^{\prime}\right)$ which is evidently the multisignature of the $\mathbf{Z}\left[\mathbf{Z}_{d}\right]$-intersection form of $W_{k}$. (For a covering $\widetilde{M} \rightarrow M$ with covering group $H$, we view the $\mathbf{Z}[H]$-intersection form on $M$ as being defined by taking intersections in $\widetilde{M}$.)

Of course $W$ itself supports an intersection form taking values in $\mathbf{Z}[G]$. Passing to $G_{k}$ corresponds to looking at intersections in $\widetilde{W_{k}}$ (where the ' ' denotes the $\mathbf{Z}_{d}$-cover). The transfer of this $G_{k}$-form to $\mathbf{Z}_{d}$ is then exactly the $\mathbf{Z}_{d}$-intersection 
form of $W_{k}$. Finally, note that completing $W_{k}$ to the branched cover $\widehat{W}_{k}$ does not change the middle dimensional homology, so it does not change the intersection form. (This requires a little work in the classical dimension.)

The difficulty with applying the realization theorem of Wall for $L_{0}(G)$ acting on a 3 -manifold, is that the surgery done to the boundary may affect the the fundamental group. In general, the most one may hope for is that the new boundary is $\mathbf{Z}[G]$ homology equivalent to the old boundary. For example, the generator of $L_{0}$ of the trivial group is an even unimodular matrix of signature 8 . The recent work of $\mathrm{A}$. Casson $[\mathbf{A M}, \mathbf{C}]$ shows that a homology sphere with Rohlin invariant 8 cannot be simply-connected. Hence if the generator acts smoothly on $S^{3}$, the fundamental group at the end will not be trivial. In the situation where one is realizing the action of $L_{0}(\mathbf{Z}[G])$ on a knot complement, it is likewise not clear that one will get back a knot in $S^{3}$ rather than some other homology sphere. However, by doing the realization carefully, we show that one can in fact get $S^{3}$.

THEOREM 1.2. Let $K$ be a knot in $S^{3}$ whose group represents to a group $G$ (so that $\sigma(K, f)$ is defined). Then for any element $A$ of $L_{0}(G)$, there is a knot $K^{\prime}$ in $S^{3}$ with $\sigma\left(K^{\prime}, f^{\prime}\right)-\sigma(K, f)=C G(A)$.

ProOF. We view an element of $L_{0}(G)$ as a matrix with values in $\mathbf{Z}[G]$. Note first that adding an element of $L_{0}(e)$ to $A$ does not change $C G(A)$, so we may assume that the ordinary signature of $A$ is zero. By performing a change of basis, in fact, we can arrange that $\varepsilon(A)$ is a standard kernel (sum of hyperbolic planes), where $\varepsilon$ is the augmentation of the group ring $\mathbf{Z}[G]$. (That this can be done follows from the fact that every invertible integer matrix is a product of elementary matrices, and hence is the augmentation of an invertible matrix over $\mathbf{Z}[G]$.) Wall's procedure for realizing $A$ amounts to the following in this dimension: take a collection of trivial circles in $X$, lying in a ball. An entry $A_{i j}$ in $A$ can be written as a sum of elements of $G$ (perhaps repeated) with coefficients \pm 1 . For each element $g$ of $G$ appearing in the sum, drag a small arc on circle $i$ around $g$ to circle $j$, and then make a small clasp around circle $j$; the sign of the clasp is determined by the sign with which $g$ appears. Adding handles along the resulting link gives a 4-manifold with intersection form given by $A$.

The key point in our context is that the loops in $X$ are, of course, homotopically trivial in the three-sphere, and so one might hope to do the dragging around in such a way that if one puts the knot $K$ back in, one has done a surgery which does not change the ambient space $S^{3}$. That this can be done follows in a straightforward manner from the following lemma.

LEMMA 1.3. Let $\{g\}$ be a collection of elements of the fundamental group of $S^{3}-K$, with some $B^{3}$ viewed as the basepoint. Then the whole collection can be represented by a link which is unlinked in $S^{3}$.

The lemma may be proved by representing $\{g\}$ by any link, and then changing crossings to obtain an unlink, along paths which miss $K$. Now the matrix $A$ may be viewed as a standard kernel, plus a matrix $B$ with $\varepsilon(B)=0$. In some ball $B^{3}$ (which will also serve as basepoint), put a bunch of copies of the 0-framed Hopf link, one for each hyperbolic plane.

Surgery on this link evidently results in just $S^{3}$ on the boundary; we will use Lemma 1.3 to produce a link isotopic to it (in $S^{3}$ ) such that adding handles realizes 


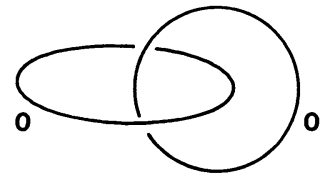

FIGURE 1

$A$. Let $b$ be a typical entry $B_{i j}$; since $\varepsilon(b)$ is trivial, $b=\Sigma \pm g$ where the total number of plus and minus signs is the same. We need to drag arcs of circle $i$ around the loops $g$ to circle $j$ and make the clasps as determined by the \pm signs. By the lemma, realize the collection of homotopy classes $\{g\}$ by a link which is trivial in $S^{3}$, meeting the $B^{3}$ in a collection of unlinked arcs. Pair up the $g$ 's so that there is $\mathrm{a}+$ and $\mathrm{a}-$ in each pair. Do an isotopy of circle $i$ so that an arc of it lies near circle $j$, and do another isotopy of circle $i$ for each pair $\{+g,-h\}$ to get the situation in Figure 2 below. Finally, break the bands in Figure 2, and insert bands going around circles in the link representing $g$ and $h$. This can be done between circles $i$ and $j$ for $i \neq j$, and between a circle and itself for $i=j$. The link which results from this process has the property of being isotopic to the link in Figure 1 when $K$ is filled back in. Adding handles along this link hence provides a cobordism between knot complements with intersection form represented by $A$.
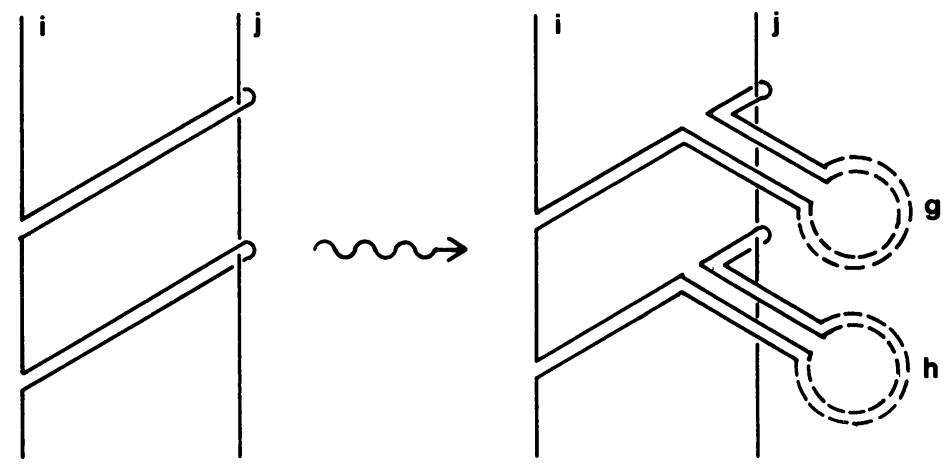

\section{FIGURE 2}

In order to use Theorem 1.1 to construct knots with interesting properties, we need to see that the algebraic map $C G: L_{2 n}(G) \rightarrow L_{2 n}\left(\mathbf{Z}_{d}\right)$ assumes many nontrivial values. This is made easier by the fact that both of these groups are easily computed. In fact, the groups are related to each other by the exact sequence due to Farrell and Hsiang on the top row of the following commutative diagram:

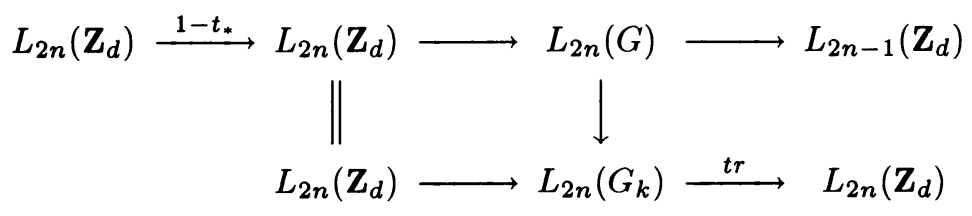

Now $L_{2 n-1}\left(\mathbf{Z}_{d}\right)=0$, so $C G$ may be understood via the composition

$$
L_{2 n}\left(\mathbf{Z}_{d}\right) \rightarrow L_{2 n}(G) \rightarrow L_{2 n}\left(G_{k}\right) \stackrel{t r}{\longrightarrow} L_{2 n}\left(\mathbf{Z}_{d}\right)
$$


But since $\mathbf{Z}_{d}$ is a subgroup of $G_{k}$, this is the same as the composition $\operatorname{tr} \circ i$ for the subgroup $\mathbf{Z}_{d}$ of $G_{k}$. The following is a well-known computation of that composition.

LEMMA 1.4. For $x$ in $L_{2 n}\left(\mathbf{Z}_{d}\right)$, $\operatorname{tr} \circ i(x)=1+t_{*}(x)+t_{*}^{2}(x)+\cdots+t_{*}^{k-1}(x)$.

PROOF. This is evident from the interpretation of transfer as passing to a covering space. (Compare Proposition 1.1 of $[\mathbf{P}]$ and $\S 3$ of [CS2].)

Using the lemma, one can give some definite computations. For $A$ in $L_{2 n}\left(\mathbf{Z}_{d}\right)$, we write $\rho(A)=\Sigma a_{j}\left(\chi^{j}+(-1)^{n} \chi^{-j}\right)$ as an element of the representation ring $R$. According to Wall, $[\mathbf{W}] \rho$ is an injection (on the kernel of the Arf-invariant, for $n$ odd) and the image contains $4 \mathbf{Z}\left[\chi+(-1)^{n} \chi^{-1}\right]$.

EXAMPLE 1.5. Let $k=2$; then $t$ acts on $\mathbf{Z}_{d}$ by multiplication by -1 , and $G_{k}$ is a dihedral group. If $n$ is even, then $t_{*}$ is the identity, so $\operatorname{tr} \circ i_{*}$ is just multiplication by 2 . However, if $n$ is odd, $t_{*}=-i d$, so the Casson-Gordon invariant in this case will be trivial.

In [R1], various knots $K(J)$ were constructed and their $C G$-invariants computed as being essentially the $q / d$-signatures $\sigma_{q / d}(J)+\sigma_{q / d}(J)$. L. Smolinsky pointed out that in dimensions $4 n+1$, our computation really showed that the + should be a -, and so the invariants should be zero. This is explained by the computation above as these were $C G$-invariants associated to dihedral covers. To get knots in dimension $4 n+1$ with nontrivial $C G$-invariants, we must use different covers.

EXAMPLE 1.6. Let $k=3$ and $d=7$, and let $t$ act on $\mathbf{Z}_{7}$ by multiplication by 2. For $n$ odd, $t_{*}\left(x^{j}-x^{-j}\right)=x^{2 j}-x^{-2 j}$, so

$$
\left(1+t_{*}+t_{*}^{2}\right)\left(x-x^{-1}\right)=\left(x-x^{-1}\right)+\left(x^{2}-x^{-2}\right)+\left(x^{4}-x^{-4}\right)
$$

which is nontrivial in $R$. It follows that there are infinitely many nontrivial values of $C G(x)$ for $x$ in $L_{2}(G)$. More generally, if $p$ is a prime congruent to 1 modulo 6 , then there is an integer $a \not \equiv 1(\bmod p)$ which satisfies $a^{3} \equiv 1(\bmod p)$. This follows from the fact $[\mathbf{H W}]$ that -3 is a square modulo such $p$, via the quadratic formula for solving $a^{2}+a+1=0$. One can check, by the same calculation as just given, that $C G$ is nontrivial for the resulting extension of $\mathbf{Z}_{p}$ by $\mathbf{Z}$.

REMARK. L. Smolinsky [S2] has given a direct construction of knots in dimensions $4 n+1$ with nontrivial Casson-Gordon invariants (and which are therefore not doubly slice by $[\mathbf{R 1}])$. As in Example 1.6, these are $C G$-invariants associated to extensions of $\mathbf{Z}_{7}$ by $\mathbf{Z}_{3}$. It would be of interest to understand the connection between his construction, which is analogous to that in $[\mathbf{R 1}, \mathbf{S 1}$, and our surgical method.

2. Even-dimensional invariants. A fundamental principle of knot theory is that the infinite cyclic cover of a knot in $S^{n}$ has the homological properties of a manifold of dimension $n-1$, and in particular satisfies Poincaré duality with coefficients in a field as a manifold of that dimension $[\mathbf{M}]$. In the author's thesis [R1], it was shown that a knot in dimension $2 n$ has, in some circumstances, $\alpha$-invariants as if it were a $(2 n-1)$-manifold, furthering this principle. The approach taken in that paper was to make the knot the boundary of an appropriate slice disk and to use the signatures of the intersection pairing of the infinite cyclic cover of the disk complement (thought of as a $2 n$-manifold). In all applications, this situation arose, but it is not clear if every knot can be chosen to be the slice of a suitable disk. For this reason, we give a new definition in terms of Seifert surfaces of $K$. 
Recall that a knot $K$ in $S^{2 n}$ bounds a $2 n-1$ manifold $V_{0}^{2 n-1}$, and that any such $V_{0}$ lifts to $X_{\infty}$, the $\mathbf{Z}$-cover of the exterior, $X$, of $K$. A compact $2 n-1$ manifold $M^{2 n-1}$ and a homomorphism $\varphi: H_{1}(M) \rightarrow \mathbf{Z}_{d}$ define a covering space $\widetilde{M}$ and an action of $\mathbf{Z}_{d}$ on $\widetilde{M}$. Atiyah and Singer $[\mathbf{A S}]$ define an invariant $\alpha\left(\widetilde{M}, \mathbf{Z}_{d}\right)$, which Wall $[\mathbf{W}]$ interprets as an element of $\mathbf{Q} R\left(\mathbf{Z}_{d}\right)$, and for which we shall use the notation $\alpha(M, \varphi)$. Let $V_{0}$ be a Seifert surface for $K$, and $i: V_{0} \rightarrow X_{\infty}$ be a lifting; write $V$ for $V_{0} \cup B^{2 n-1}$.

THEOREM 2.1. (i) For any $d, \alpha(V, \varphi \circ i)$ is well defined as an element of $\mathbf{Q} R\left(\mathbf{Z}_{d}\right) / R\left(\mathbf{Z}_{d}\right)$.

(ii) If $d$ is a power of a prime $p$, then $\alpha(V, \varphi \circ i)$ is independent of the choice of Seifert surface and hence is well defined as an element of $\mathbf{Q} R\left(\mathbf{Z}_{d}\right)$.

As an immediate corollary, we see that the following are good definitions.

DEFINITION 2.2. For $d$ a prime-power, and $\varphi: H_{1}\left(X_{\infty}\right) \rightarrow \mathbf{Z}_{d}$, define

$$
\alpha(K, \varphi)=\alpha(V, \varphi \circ i) \in \mathbf{Q} R .
$$

Otherwise, define $\alpha(K, \varphi)=\alpha(V, \varphi \circ i) \in \mathbf{Q} R / R$.

PROOF OF THEOREM 2.1. It suffices to consider $\varphi$ which are onto. Also, it is convenient to work in $Y_{\infty}$, the $\mathbf{Z}$-cover of surgery on $K$, to which $V=V_{0} \cup$ $B^{2 n-1}$ lifts. As in [CG, R1, Lemmas 4.1-4.6], $Y_{\infty}$ satisfies Poincaré duality with coefficients in $\mathbf{Q}$. In case (ii), where $d=p^{r}, \widetilde{Y}_{\infty}$, the induced $p^{r}$-fold cover, also satisfies Poincaré duality over $\mathbf{Q}$. The (Q-)fundamental class of $Y_{\infty}$ is carried by $V$; this implies (cf. Lemma $4.6 \mathrm{~b}$ of [R1]) that $\varphi \circ i_{*}: H_{1}(V) \rightarrow \mathbf{Z}_{d}$ is onto, even in the case when $d$ is composite. Hence, in case (ii) $V$ is connected and carries the fundamental class of $\widetilde{Y}_{\infty}$. These statements may be summarized (in case ii) by saying that $Y_{\infty}$ satisfies $2 n-1$ dimensional Poincaré duality with coefficients in $\mathbf{Q}\left[\mathbf{Z}_{p^{r}}\right]$, and $V$ carries the $\mathbf{Q}\left[\mathbf{Z}_{p^{r}}\right]$ fundamental class, and that $H_{*}\left(V ; \mathbf{Q}\left[\mathbf{Z}_{p^{r}}\right]\right) \rightarrow$ $H_{*}\left(Y_{\infty} ; \mathbf{Q}\left[\mathbf{Z}_{p^{r}}\right]\right)$ is onto.

To show that $\alpha(V, \varphi \circ i)=\alpha(W, \varphi \circ j)$ (in the appropriate ring) for Seifert surfaces $V$ and $W$, we may assume that $i(V)$ and $j(W)$ are disjoint, by taking different lifts if necessary. Since $V$ and $W$ both carry $\left[Y_{\infty}\right]$, they cobound a compact $2 n$-manifold $A$. See Figure 3 below.

Claim. (1) $H_{1}(A) \rightarrow \mathbf{Z}_{d}$ is onto.

(2) $H_{*}(V) \oplus H_{*}(W) \rightarrow H_{*}(A)$ is onto in case (ii); coefficients being taken in $\mathrm{Q}\left[\mathbf{Z}_{p^{r}}\right]$.

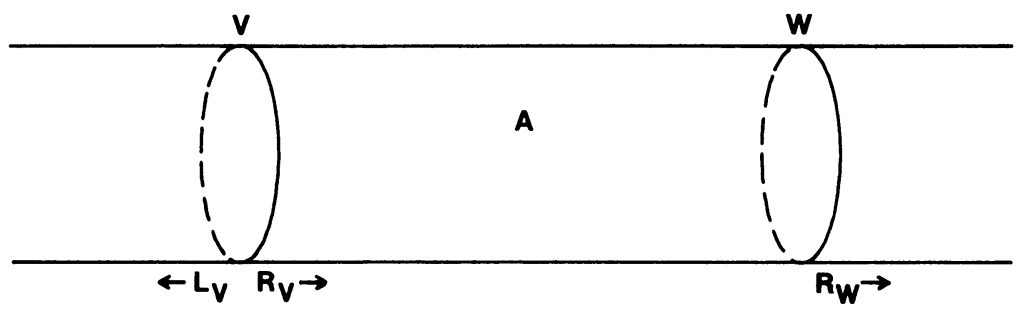


For part (1), note that $\widetilde{A}$, the induced $p^{r}$-fold cover of $A$, is connected, because $\widetilde{Y_{\infty}}, \widetilde{V}$, and $\widetilde{W}$ are connected. Hence one can use $A$ to compute the difference $\alpha(V, \varphi \circ i)-\alpha(W, \varphi \circ j)$ to be $\rho(A, \varphi)$.

Part (2) is shown by the excision argument which follows: All coefficients will be taken in $\mathbf{Q}\left[\mathbf{Z}_{p^{r}}\right]$ for the rest of the argument. Let $R_{V}$ be the component of $Y_{\infty}-V$ in which $W$ lies and $R_{W}$ the component of $Y_{\infty}-W$ in which $V$ does not lie, and let $L_{V}$ be the complement of $R_{V}$. ( $R$ and $L$ stand for 'right of' and 'left of' in Figure 3.) By excision, the inclusion $\left(R_{V}, V\right) \rightarrow\left(Y_{\infty}, L_{V}\right)$ is an isomorphism on homology; since it factors through $\left(Y_{\infty}, V\right), H_{j}\left(R_{V}, V\right) \rightarrow H_{j}\left(Y_{\infty}, V\right)$ is an injection. Comparing the long exact sequence of $\left(R_{V}, V\right)$ with that of $\left(Y_{\infty}, V\right)$, and noting that $H_{j}(V) \rightarrow H_{j}\left(Y_{\infty}\right)$ is onto, we get that $H_{j}\left(R_{V}\right) \rightarrow H_{j}\left(R_{V}, V\right)$ is the zero map. Finally, consider the following diagram:

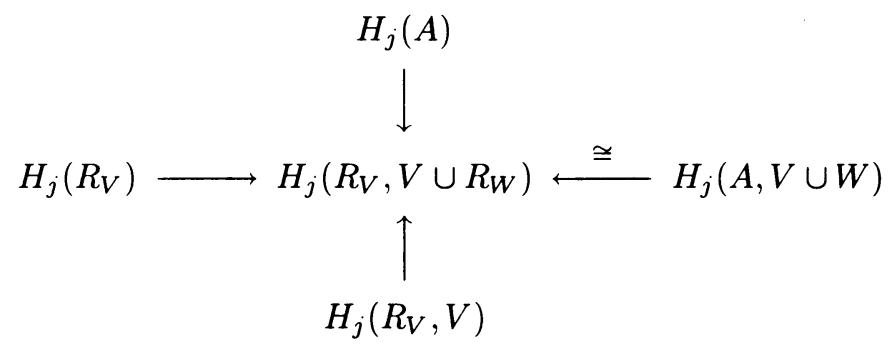

All maps are inclusions, and the rightmost one is an isomorphism, again by excision. A diagram chase shows that $H_{j}(A) \rightarrow H_{j}(A, V \cup W)$ is trivial, so that $H_{j}(V \cup W) \rightarrow$ $H_{j}(A)$ is onto.

To prove the theorem, we use the fact that the coefficients of the $\chi^{j}$ in $\rho(A)$ can be interpreted as eigenspace signatures of the $\mathbf{Z}\left[\mathbf{Z}_{p^{r}}\right]$ valued intersection form of $A$. But since the homology of $A$ is carried in the boundary of $A$, all intersections (even over $\left.\mathbf{Z}\left[\mathbf{Z}_{p^{r}}\right]\right)$ must be trivial. Hence $\alpha(V, \varphi \circ i)=\alpha(W, \varphi \circ j)$ as claimed.

It is definitely not true that Theorem 2.1 can be extended to say that $\alpha(V, \varphi \circ i)$ is well defined in $\mathbf{Q} R\left(\mathbf{Z}_{d}\right)$ for $d$ composite. This is because of a theorem of $\mathrm{S}$. Cappell and the author.

THEOREM 2.3 [CR]. Let $L$ be a lens space of dimension $\geq 5$ such that $\pi_{1}(L)$ is of composite order. Then there is a homotopy lens space $L^{\prime}$, which is (integral) homology cobordant to $L$, but for which $\alpha(L, \varphi) \neq \alpha\left(L^{\prime}, \varphi^{\prime}\right)\left(\varphi\right.$ and $\varphi^{\prime}$ are isomorphisms of $\pi_{1}$ with $\mathbf{Z}_{d}$ which extend over the homology cobordism.)

COROLLARY 2.4. In every even dimension (above 4) there are infinitely many knots which admit Seifert surfaces with differing $\alpha$-invariants.

PROOF. In the author's paper [R2], it is shown that there is a fibered knot in $S^{2 k}$ whose fiber is a punctured lens space with $\left|\pi_{1}\right|=d$, whenever one can solve the equation

$$
c^{k} \equiv 1 \quad(\bmod d), \quad c^{j} \not \equiv 1 \quad(\bmod d) \quad \text { for } j<k .
$$

By Dirichlet's theorem on primes in an arithmetic progression, there are an infinite number of primes of the form $n k+1$. Recall that for $p$ a prime, the group of units in $\mathbf{Z}_{p}$ is cyclic of order $p-1$. Hence if $p$ is an odd prime of the form $n k+1$, there is an element $\alpha$ of $\mathbf{Z}_{p}^{*}$ of order exactly $k$ in $\mathbf{Z}_{p}^{*}$. Let $p$ and $q$ be primes of this form, 
and choose $\alpha$ and $\beta$ of order $k$ in $\mathbf{Z}_{p}^{*}$ and $\mathbf{Z}_{q}^{*}$, respectively. The Chinese remainder theorem says that there is an integer $\gamma$ congruent to $\alpha(\bmod p)$ and to $\beta(\bmod q)$; it follows that $\gamma$ is a solution to the above equation for $d=p q$.

Now take a knot with fiber a punctured lens space as provided by the theorem of [R2]. By Theorem 2.3, there is a homology cobordism from $L$ to a homotopy lens space $L^{\prime}$ with $\pi_{1}=\mathbf{Z}_{d}$. If one doubles the cobordism along $L^{\prime}$ and inserts this new homology cobordism into the knot complement, one obtains a knot (no longer fibered) with Seifert surfaces $L$ and $L^{\prime}$ with different $\alpha$-invariants.

3. Applications to odd-dimensional knots. The main applications we have in mind for the invariants defined in the previous two sections is to the questions of which knots are doubly slice, and which doubly slice knots are in fact the double of a disk knot. As our knots will be gotten via the action of surgery groups, we will need some model knots whose fundamental groups are the extensions discussed previously.

PROPOSITION 3.1. Let $G$ be an extension $\mathbf{Z} \times_{t} \mathbf{Z}_{d}$, where $t$ acts by $t(g)=g^{r}$ and both $r$ and $r-1$ are units modulo $d$. Then $G$ is the group of a doubled disk knot in $S^{n}$ for $n \geq 6$.

ProOF. The homology of $G$ may be computed via a Mayer-Vietoris sequence:

$$
H_{j}\left(\mathbf{Z}_{d}\right) \stackrel{1-t_{*}}{\longrightarrow} H_{j}\left(\mathbf{Z}_{d}\right) \rightarrow H_{j}(G) \rightarrow H_{j-1}\left(\mathbf{Z}_{d}\right)
$$

to be $\mathbf{Z}$ in dimension 1 and zero in dimension 2 . Also, it is easily seen that a generator of the $\mathbf{Z}$ normally generates $G$. Kervaire's construction $[\mathbf{K}]$ shows how to build a disk pair in dimension 6 or more with $\pi_{1}$ of the complement equal to $G$, and such that the knot on the boundary also has group $G$. Doubling this disk pair results in the desired knot.

Kervaire's procedure works well to produce knotted spheres in $S^{5}$ with a given group, and hence, by removing an unknotted ball pair, to produce knotted disks as well. Unfortunately, doubling such a disk knot results in a fundamental group which is too large, and to get the knot on the boundary to have the same fundamental group as the disk knot requires one to do a surgery on a 4-manifold, which one does not know how to do. However, we can reproduce Proposition 3.1 for certain extensions.

PROPOSITION 3.2. Let $G$ be an extension $\mathbf{Z} \times{ }_{t} \mathbf{Z}_{d}$, where $d$ is of the form $d=3 n^{2}+3 n+1$ and $t$ acts by $t(g)=g^{3 n+1}$. Then $G$ is the group of a doubled disk knot in $S^{5}$.

ProOF. Consider the ribbon disk $D_{n}$ in $B^{4}$ drawn in Figure 4(a); its complement has the handlebody decomposition drawn in Figure 4(b) with two 1-handles and a single 2-handle.

From Figure 4, one immediately reads off the presentation

$$
\pi_{1}\left(B^{4} \backslash D_{n}\right)=\left\{x, y:\left(x y^{-1}\right)^{n+1}\left(x^{-1} y\right)^{n}\right\} .
$$

Here $x$ and $y$ are meridians and are represented by the cores of the 1-handles. Making a substitution $z=x y^{-1}$ makes the relation

$$
z^{n+1} x^{-1} z^{-n} x
$$




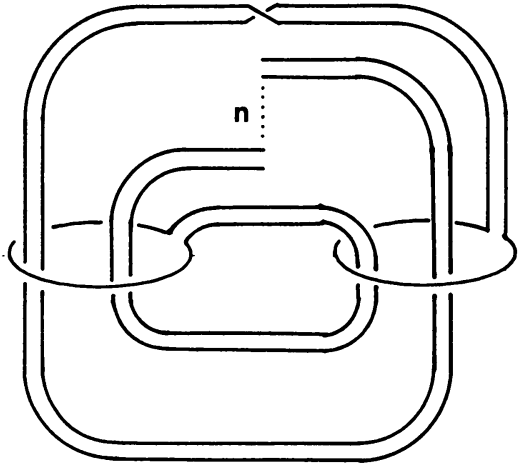

(a)
FIGURE 4

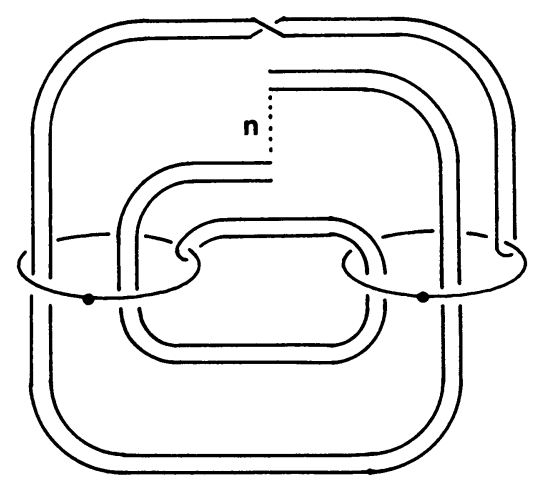

(b)

Let $\Delta_{n}$ be the disk knot in $B^{5}$ gotten by 3 -twist spinning the knot $D_{n}$; then just as in $[\mathbf{Z}]$, the complement is fibered, with fiber the 3-fold branched cover of $D_{n}$. To compute the fundamental group of this branched cover, we use the ReidemeisterSchreier theorem [MKS], which yields a presentation of the unbranched cover, and by killing a lift of a meridian, a presentation of the group of the branched cover. The result is

$$
\left\{u, v, w: u^{n+1}=v^{n}, v^{n+1}=w^{n}, w^{n+1}=u^{n}\right\} .
$$

This group is abelian: raise both sides of the relation $u^{n+1}=v^{n}$ to the $(n+1)$ st power, obtaining $\left(u^{n}\right)^{n+1} u=\left(v^{n+1}\right)^{n}$, which is the same (using the other two relations) as $w^{(n+1)^{2}} u=w^{n^{2}}$. Hence $u$ is a power of $w$, and likewise $v$ is a power of $u$, so that $v$ is also a power of $w$ and the group is cyclic. The covering translation acts, with respect to this presentation, by sending $u \rightarrow v \rightarrow w$.

It is now an easy exercise to show that the group is cyclic of order $3 n^{2}+3 n+1$, and that the covering translation acts as stated in the statement of the proposition. Finally, recall that the group of a ribbon knot surjects onto the group of its associated ribbon disk. Thus the knot on the boundary of the 3-twist spun disk $\Delta_{n}$, which is itself a twist-spun knot, has group which surjects onto the group of $\Delta_{n}$. As in Proposition 3.1, doubling $\Delta_{n}$ produces the desired knot in $S^{5}$.

REMARK. Levine has shown that doubled disk knots are doubly slice, so these propositions provide doubly slice knots as well.

THEOREM 3.3. There are infinitely many knots in any odd dimensional sphere $S^{2 n-1}$ which are algebraically doubly slice but not doubly slice. Further, in all but the classical dimension, these may be chosen to have exteriors which are homotopy equivalent (rel boundary, preserving meridians), to one another and to a doubly slice knot. In the classical case, they have the same $\mathbf{Z}[\mathbf{Z}]$-homology type.

PROOF. In dimensions above the classical one, we let $G$ be an extension $\mathbf{Z} \times{ }_{t} \mathbf{Z}_{d}$ as above. If $n$ is even, $d$ can be any odd prime, and $t=$ multiplication by -1 . If $n$ is odd, we use $d=7$, and let $t$ be multiplication by 2 . (Actually, for $n>2$, any prime congruent to $1(\bmod 6)$ would do-- $t$ would then be multiplication by $a$ where $a^{3}=1(\bmod d)$ ). Proposition 3.1 (or 3.2 -note that $\left.7=3+3+1\right)$ shows that there is a doubly slice knot $K$ (in fact a doubled disk knot) in dimension $2 n-1$ with 
group $G$. The computation of the homomorphism $C G$ in the two examples given above shows that there infinitely many elements of $L_{2 n}(G)$ with $C G$ all different. Letting these elements act $[\mathbf{W}]$ on the exterior of the knot $K$ provides the knots for the theorem. To verify that they are not doubly slice, we use Theorem 2.2 of [R1], which implies that the Casson-Gordon invariants are bounded by the dimension of the middle dimensional homology of the branched cover which define them. Since the complement of the $K$ is built by doubling a manifold which is effectively a 2complex, $K$ has the property that its 2 (resp. 3 ) fold branched cover has no middle dimensional homology. If the knots were doubly slice, the Casson-Gordon invariants would have to vanish, which would be a contradiction. In the classical dimension, we use any knot with a dihedral representation (for example a 2-bridge knot) and let $L_{0}(G)$ act on its exterior. Theorem 1.2 shows how to do this to get a knot in $S^{3}$.

In his thesis [S1], L. Smolinsky gave examples of doubly slice knots in $4 k-1$ dimensional spheres which are not doubled disk knots, by using the Casson-Gordon invariants associated to certain carefully specified covering spaces. His method works for knots in dimensions $4 k+1$, but he did not find any knots with nontrivial invariants, essentially because of Example 1.5. (He has found such examples more recently - see the remark after Example 1.6.) From our point of view, however, such examples are easily found with the aid of the computation of $C G$ for 3-fold covers (Example 1.6). Moreover, we can construct such knots with the additional property of having complements homotopy equivalent to the complement of a doubled disk knot. In order to make this refinement of Smolinsky's result we use the fact, due to Cappell and the author, that the natural map $L_{2 n}\left(\mathbf{Z}_{p q}\right) \rightarrow \Gamma_{2 n}\left(\mathbf{Z}\left[\mathbf{Z}_{p q}\right] \rightarrow \mathbf{Z}\right)$ has a large kernel. (This fact underlies Theorem 2.3.) In fact, we have

THEOREM 3.4 [CR]. Let $p$ and $q$ be different odd primes. Then any element in $L_{2 n}\left(\mathbf{Z}_{p q}\right)$ whose multisignature is of the form $4 k\left(\chi+(-1)^{n} \chi^{-1}\right)$ and whose simplyconnected signature or Arf-invariant vanishes, is in the kernel of the natural map $L_{2 n}\left(\mathbf{Z}_{p q}\right) \rightarrow \Gamma_{2 n}\left(\mathbf{Z}\left[\mathbf{Z}_{p q}\right] \rightarrow \mathbf{Z}\right)$.

Using this, we get

THEOREM 3.5. In all odd dimensions, there are infinitely many knots which are homotopy equivalent (rel boundary) to a doubled disk knot, are doubly slice, but are not themselves doubled disk knots.

PrOOF. As in the Theorem 3.3, the groups involved are slightly different for $n$ even and $n$ odd. For $n$ even, any primes will do, while for $n$ odd, choose $p$ and $q$ to be different primes congruent to $1 \bmod 6$. Choose extensions of $\mathbf{Z}$ by $\mathbf{Z}_{p q}$ also as in the Theorem 3.3. As in that theorem, there is a doubled disk knot in $S^{2 n-1}$ whose complement has fundamental group $G$. Now our calculation of the homomorphism $C G$ shows that for an element $a_{k}^{\prime}$ of $L_{2 n}\left(\mathbf{Z}_{p q}\right)$ with multisignature $4 k\left(\chi+(-1)^{n} \chi^{-1}\right)$, we have that $C G\left(a_{k}\right)$ is nontrivial as an element of $R_{p q}$. Hence Smolinsky's theorem [S1] can be applied to show that the knots gotten by realizing the action of the image of $a_{k}$ in $L_{2 n}(G)$ on the exterior of $K$ cannot be doubled disk knots.

In order to see that these knots will be doubly slice, recall that the unknot is characterized (in dimensions other than 4) as having complement homotopy equivalent to the circle. It is easily seen that this is equivalent to having $\pi_{1}=\mathbf{Z}$ and 
trivial $\mathbf{Z}[\mathbf{Z}]$-homology. Consider the following diagram:

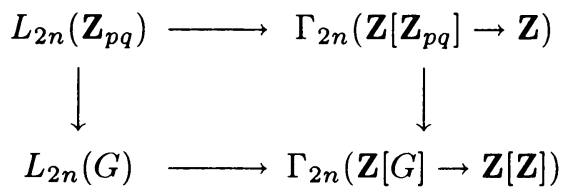

By Theorem 3.4, $a_{k}$ is in the kernel of the top map, its image in $L_{2 n}(G)$ dies in $\Gamma_{2 n}(\mathbf{Z}[G] \rightarrow \mathbf{Z}[\mathbf{Z}])$. Hence a cobordism carrying $a_{k}$ may be surgered to be a $\mathbf{Z}[\mathbf{Z}]$ homology cobordism, with fundamental group $G$. Double this cobordism along $X_{k}$, to obtain a $\mathbf{Z}[\mathbf{Z}]$-homology cobordism from $X$ to itself with fundamental group $G$. Now $K$, being a doubled disk knot, is doubly slice. If one inserts the $\mathbf{Z}[\mathbf{Z}]$-homology cobordism into the unknot of which $K$ is a slice, one gets an unknot with $K_{k}$ as a slice.

4. Applications to even-dimensional knots. Our original motivation for defining the even-dimensional Casson-Gordon invariants was to provide an obstruction to a knot being doubly slice, and thereby to show the existence, in all dimensions, of non-doubly slice knots which satisfied an algebraic condition which we called "algebraically doubly slice". In attempting to prove that the vanishing of the invariants we used was sufficient to make a knot doubly slice, we established Theorem 4.5 below, which states that an even-dimensional knot which has complement homology equivalent to a doubly slice knot is itself doubly slice. It was immediately clear to the author that this contradicts Theorems 4.12-4.16 of [R1], and in fact these theorems are incorrect. (The mistake turns out to be in the use of the universal coefficient theorem for noncompact spaces: by Smith-theory, certain spaces involved in the proof of those theorem are seen to have trivial $\mathbf{Z}_{d}$-homology, but this does not imply that they have trivial rational homology, which is what is needed to make their $C G$-invariants vanish.)

However, using the $\mathbf{Q} / \mathbf{Z}$-valued Casson-Gordon invariants defined in $\S 2$, we are able to recover the main result of [R1, Theorem 4.17], which gives non-doubly slice knots in all dimensions which are algebraically doubly slice. The theorem which leads to obstructions to double null-concordance (replacing $4.12-4.16$ of [R1]) is the following:

THEOREM 4.1. Let $K$ be a knot in $S^{2 n}$, and suppose $K$ is doubly slice. Then $H_{*}\left(X_{\infty}\right)=G_{*} \oplus G_{*}^{\prime}$, where (1) $G_{k-1}=G_{k-1}^{\prime}$, (2) The linking form vanishes on both $G_{k-1}$ and $G_{k-1}^{\prime}$, and for all surjections $\varphi: H_{1}\left(X_{\infty}\right) \rightarrow \mathbf{Z}_{d}$ with $\varphi_{*}$ vanishing on $G_{*}$ or $G_{*}^{\prime}$, we have that the $\mathbf{Q} / \mathbf{Z}$-valued Casson-Gordon invariant $\alpha(K, \varphi)=0$.

ProOF. The splitting of $H_{*}\left(X_{\infty}\right)$ results, as usual, from a Mayer-Vietoris sequence for the homology of the infinite cyclic cover of the complement of the unknot as split up by $X_{\infty}$. For convenience, let $Y$ be the result of surgery on $S^{2 n}$ along $K$, and let $Y_{\infty}$ be its infinite cyclic cover. Then in particular, $G_{*}$ is the kernel of the inclusion of $Y_{\infty}$ into $A_{\infty}$, which is the infinite cyclic cover of the complement of one of the discs into which $K$ splits the unknot. If $\varphi$ is a homomorphism as stated in the theorem, then by hypothesis, it extends to a homomorphism on $H_{1}\left(A_{\infty}\right)$.

Now let $W^{2 n}$ be a section of $A_{\infty}$, i.e. a codimension-one submanifold separating the ends of $A_{\infty}$, and such that $V=\partial W$ is the lift of a Seifert surface for $K$ (capped off under the surgery which produces $Y$ ). As in the proof of Theorem 2.1, the 
composition of (the extension of) $\varphi$ with the inclusion of $W$ in $A_{\infty}$ is surjective. This means that we can use the multisignatures of the intersection form on $\widetilde{W}$ to compute $\alpha(V, \varphi)$ which is, by definition $\alpha(K, \varphi)$. Hence the coefficients in $\alpha(K, \varphi)$ are all integral, i.e. it is trivial in $\mathbf{Q} R\left(\mathbf{Z}_{d}\right) / R\left(\mathbf{Z}_{d}\right)$.

As promised above, this leads to non-doubly slice knots in all even dimensions. To see this, we make use of the examples of knots constructed in [R2] and used in Corollary 2.4. As shown in the proof of that corollary, for every $k$, there is a prime $p$, and a $(\bmod p)$ unit $c$ so that $c^{k} \equiv 1(\bmod d), c^{j} \not \equiv 1(\bmod d)$ for $j<k$. Such a number $c$ determines a fibered knot $K$ whose fiber is a punctured lens space $L_{0}$ with $\pi_{1}(L)=\mathbf{Z}_{p}$. The monodromy of the fibering induces multiplication by $c$ on $H_{1}(L)$; and therefore by $c^{j}$ on $H_{2 j-1}(L)$.

LEMMA 4.2. Let $L$ be any lens space, and $\varphi$ any isomorphism of $H_{1}(L)$ with $\mathbf{Z}_{d}$, where $d$ is any odd number. Then $\alpha(L, \varphi)$ is a nonintegral element of $\mathbf{Q} R\left(\mathbf{Z}_{d}\right)$.

PROOF. The action of $\mathbf{Z}_{d}$ on $S^{2 n-1}$ may be viewed as the join of $n$ standard rotations of $S^{1}$ by angles $2 \pi / q_{j}$, so its alpha-invariant is the product of the alphainvariant of these rotations, each of which is given by an expression of the form

$$
\alpha_{j}=\frac{1+\chi^{q_{j}}}{1-\chi^{q_{j}}} .
$$

It is easy to see, by direct calculation, that such an $\alpha_{j}$ will not be integral. Now assume, inductively, that the lemma is true for lens spaces of dimension less than $2 n-1$. Since the action on $S^{2 n-1}$ is a join with an action on $S^{1}$, it follows that

$$
\alpha(L)=\alpha\left(L^{\prime}\right) \frac{1+\chi^{r}}{1-\chi^{r}}
$$

for some $(2 n-3)$-dimensional lens space $L^{\prime}$ and some number $r$. (We have suppressed the $\varphi^{\prime}$ 's since they just permute the $\chi^{j}$ 's around and do not affect the integrality.) But $1+\chi^{r}$ has an (integral) inverse in $R \mathbf{Z}_{d}$, given by $\sum_{j=0}^{d-1} \chi^{2 r j}$. If $\alpha(L)$ is integral, then, it follows that $\alpha\left(L^{\prime}\right)$ is integral as well, contradicting the inductive hypothesis.

DEFINITION 4.3 [R1]. A knot in $S^{2 n}$ is said to be algebraically doubly slice if the linking form $[\mathbf{L}]$ is hyperbolic.

It was shown by Stoltzfus [St] that a doubly slice knot was algebraically doubly slice in this sense.

THEOREM 4.4. For all $n>1$, there are non-doubly slice knots in $S^{2 n}$ which are algebraically doubly slice.

PROOF. For $n=2$, this is shown in [R1, 4.10, 4.11], using the $\mu$-invariant of a knot in $S^{4}$. For higher values of $n$, we use the fibered knots $K$ constructed above with lens space fibers, and fundamental group $\mathbf{Z}_{p}$ for $p$ a prime; as remarked above there are such knots in every even dimension. For $n$ odd, there is no linking form, and so the theorem follows from Theorem 4.1 using Lemma 4.2. For $n$ even, the linking form of $K$ is given by a $(1 \times 1)$ matrix $(1 / p)$, with action of the covering translation given by $c^{n-1}$. Now according to Levine [L], there is a simple knot $K^{\prime}$ in $S^{2 n}$ with the same linking form as $K$ (for $n>2$ ). It is evident that $K \#-K^{\prime}$ is algebraically doubly slice; we claim that it is not doubly slice. To see this, of 
course, we use Theorem 4.1: Since $p$ is a prime, there is only one way to decompose $H_{1}(L)=\mathbf{Z}_{p}$ as a direct sum, i.e. as $\mathbf{Z}_{p} \oplus 0$. There is an obvious homomorphism $\varphi$ (vanishing on the 0 -summand!) to $\mathbf{Z}_{p}$. Suppose now that $K \#-K^{\prime}$ is doubly slice. Theorem 4.1 implies that $\alpha\left(K \#-K^{\prime}, \varphi\right)=\alpha(L, \varphi)$ must then be rational-but this contradicts Lemma 4.2 .

The theorems proved in $\S 3$ demonstrated that an odd-dimensional knot with complement homotopy equivalent to that of a doubly slice knot need not itself be doubly slice. A similar result pertained to doubled disk knots. Both of these facts stand in sharp contrast to the case of ordinary knot concordance, where the homotopy type determines the the concordance class. The situation is different for even-dimensional knots: the next theorem demonstrates that, in some sense, the property of being doubly slice depends only on the $(\mathbf{Z}[\mathbf{Z}])$-homology type of the complement of the knot. One application of this theorem is to give even-dimensional knots which are doubly slice, but not doubled disk knots. In fact, we will see that as in odd dimensions, there are knots with complements homotopy equivalent to those of doubled disk knots which are not doubled disk knots.

THEOREM 4.5. Let $K$ and $J$ are knots in $S^{2 n}$, and suppose that $J$ is doubly slice. Let $f: X(K) \rightarrow X(J)$ be a map which restricts to a homeomorphism on the boundary. For $n>2$, if $f$ induces an isomorphism of the homology groups of the infinite cyclic covers, then $K$ is doubly slice as well. For $n=2$, the same statement holds if we assume in addition that the $\mu$-invariant of $K$ (see [R1]) is trivial.

To identify doubly slice knots, we use the following lemma, due to J. Levine [L].

LEMMA 4.6. For $n>1$, a knot $\left(S^{n}, K\right)$ is doubly slice if and only if $Y(K)$ embeds in a homotopy $S^{1} \times S^{n}$, separating, and inducing an isomorphism on $H_{1}$.

PROOF OF THEOREM 4.5. Suppose that $J$ is doubly slice, and write $S^{1} \times S^{2 n}$ as $A_{1} \cup_{Y(J)} A_{2}$ as in the lemma. As $X(K)$ and $X(J)$ are parallelisable, being submanifolds of a sphere, it is easy to cover $f$ by a map of stable normal bundles. Any homeomorphism of $S^{1} \times S^{2 n-1}$ extends over $S^{1} \times D^{2 n}$, so $f$ extends to a map on $Y(K)$ which is also covered by a bundle map.

The first thing to see about this map (which we will continue to label $f$ ) is that it extends to normal maps $B_{i} \rightarrow A_{i}$. A standard transversality construction [B] says that this can be done provided the specific lifting of the Spivak normal space of $Y(J)$ corresponding to $f$ extends to $A_{i}$. The obstructions to doing this lie in $H^{j+1}\left(A_{i}, Y(J) ; \pi_{j}(G / P L)\right)$ which is trivial, unless $j=2 n$. Let $A$ be either $A_{1}$ or $A_{2}$; we drop the subscript for the rest of the proof. It is straightforward to construct a degree-one map $g$ (rel boundary) from $(A, Y(J))$ to $\left(S^{1} \times D^{2 n}, S^{1} \times S^{2 n-1}\right)$; since all stable normal bundles are trivial, $g$ (and all other maps in the rest of the proof) will be a normal map. By naturality, the obstruction to $g \circ f$ (as a map from $Y(J)$ ) being normally null-cobordant is the pull-back under $g^{*}$ of the obstruction to null-cobordism of $f$. But $g \circ f$ is null-cobordant, as $J$ is a slice knot. Further, $g^{*}$ is an isomorphism on cohomology, so $f$ is null-cobordant as well.

Now Cappell and Shaneson [CS1] have defined an obstruction to doing surgery on $B_{i}$ to obtain a homology equivalence with various coefficients. Since $f$ is assumed to be a homology equivalence over $\mathbf{Z}[\mathbf{Z}]$, the obstruction lies in a group $\left.\Gamma_{2 n+1}(\mathbf{Z} \mid \pi] \rightarrow \mathbf{Z}[\mathbf{Z}]\right)$. Here $\pi$ is the fundamental group of $A$. By definition, the $\Gamma$-group is a subgroup of the ordinary $L$-group $L_{2 n+1}(\mathbf{Z}[\mathbf{Z}])$, which is in turn iso- 
morphic to $L_{2 n}(\mathbf{Z})$ which is isomorphic to $\mathbf{Z}$ or $\mathbf{Z}_{2}$ for $n$ even or odd. Consider first the case $n$ even and greater than 2. The obstruction in $\mathbf{Z}$ may be computed by taking a submanifold $V$ of $A$ representing a generator of $H_{2 n}(A, Y(J))=\mathbf{Z}$. If $f$ is transverse to $V$, then the obstruction is $k=\sigma\left(f^{-1}(V)\right)-\sigma(V)$. Since $f$ is a $\mathbf{Z}[\mathbf{Z}]$-homology equivalence on the boundary, then this difference is divisible by 8. Hence it may be killed by removing an $S^{1} \times D^{2 n}$ representing a generator of $H_{1}(B)$, and replacing it with $S^{1} \times \#_{k} M^{2 n}$, where $M^{2 n}$ is the Milnor manifold of signature -8 . For $n=2$, this procedure works if and only if $k$ is divisible by 16 , because of Rohlin's theorem. It is easy to see that this divisibility holds if and only if $\mu(K)=0$. For $n$ odd, the same trick works, only for $M^{2 n}$ we use the Kervaire manifold of Arf invariant 1.

Since the $B_{i}$ are now (after the surgery) homology equivalent to the $A_{i}$, their union is homology equivalent to $S^{1} \times S^{2 n}$. But by [CS1], we may in fact assume that the homology equivalences $B_{i} \rightarrow A_{i}$ induce isomorphisms on the fundamental group. Moreover, the map $Y(K) \rightarrow Y(J)$ has degree one, and so induces a surjection on $\pi_{1}$. An exercise in using van Kampen's theorem now shows that $B_{1} \cup_{Y(K)} B_{2}$ has fundamental group $\mathbf{Z}$, so that it is in fact homotopy equivalent to $S^{1} \times S^{2 n}$. Therefore (by Lemma 4.6 ), $K$ is doubly slice.

One special case of the theorem is interesting, for knots in $S^{4}$ :

COROLlaRY 4.7. Let $K$ be a knot in $S^{4}$ such that $H_{1}\left(X_{\infty}\right)$ is trivial. $K$ is doubly slice if and only if $\mu(K)$ is trivial.

For the proof we merely remark that such a knot is homology equivalent to the unknot, which is certainly doubly slice. Note also that a knot which has a homology ball for a Seifert surface certainly satisfies the hypothesis of the corollary.

The knots which are constructed as boundaries of lens spaces are another interesting class to which Theorem 4.5 applies. The corollary which results is more or less a converse to Theorem 4.4 .

COROLLARY 4.8. Let $K$ and $K^{\prime}$ be fibered knots in $S^{2 n}$ with fibers punctured lens spaces $L$ and $L^{\prime}$. If $L$ is homotopy equivalent to $L^{\prime}$, and their monodromies act the same on $H_{1}$, then $K \#-K^{\prime}$ is doubly slice.

ProOF. Again we apply the theorem: For the one knot we use $K \#-K^{\prime}$, and we notice that its exterior is homotopy equivalent to that of $K \#-K$, which is doubly slice $[\mathbf{S u}]$.

As a final application of our techniques, we establish the existence of evendimensional knots which are doubly slice but are not doubled disk knots. As mentioned in the introduction, this was done in dimensions 3 and 4 by Levine [L], and in dimensions $4 k-1$ by Smolinsky [S1]; knots in $S^{4 k+1}$ are taken care of by Theorem 3.5. As in that theorem, we will again find that being a doubled disk knot is not a homotopy property. To detect doubled disk knots, we use the even-dimensional invariants to give an analogue of Smolinsky's theorem. Smolinsky has told the author that he was aware of a theorem such as Theorem 4.9 below. There is a slightly different approach to the problem which we will mention at the end of the section.

THEOREM 4.9. Let $K$ be a knot in $S^{2 n}$ which is the double of a disk knot $\Delta$ in $B^{2 n}$. If $X_{\infty}$ is the infinite cyclic cover of the exterior $X$ of $K$, then $H_{1}\left(X_{\infty}\right)=$ $G \oplus G^{\prime}$, where there is a surjection $G \rightarrow G^{\prime}$. Further, if $\varphi: H_{1}\left(X_{\infty}\right) \rightarrow \mathbf{Z}_{d}$ has $\varphi(G)=0$, then $\alpha(K, \varphi)=0$ (in the appropriate ring for it to be defined.) 
PROOF. Recall that Levine [ $\mathbf{L}]$ shows that a doubled disk knot is doubly slice by exhibiting it as a cross section of the unknot, where one of the disks into which $k$ divides the unknot is simply the spun disk $\Sigma \Delta=\Delta \times \mathbf{I}$. Letting $A$ be the exterior of $\Sigma \Delta$, and $A^{\prime}$ be the exterior of the other disk (really a '1-twist spin' of $\Delta$ ), we have that $H_{1}\left(X_{\infty}\right) \cong G \oplus G^{\prime}$, where $G$ is defined to be $\operatorname{ker}\left[H_{1}\left(X_{\infty}\right) \rightarrow H_{1}\left(A_{\infty}\right)\right]$ and $G^{\prime}$ is defined to be $\operatorname{ker}\left[H_{1}\left(X_{\infty}\right) \rightarrow H_{1}\left(A_{\infty}^{\prime}\right)\right]$. The usual Mayer-Vietoris argument [GL, R1, S1] shows that $H_{1}\left(X_{\infty}\right)$ splits as claimed; following [S1] one shows that $G$ surjects onto $G^{\prime}$.

Now any Seifert surface for $K$ is the boundary of a relative Seifert surface for the disk knot $\Sigma \Delta$, and that relative Seifert surface lifts up to $A_{\infty}$. If $\varphi(G)=0$, it extends to a homomorphism on $H_{1}\left(A_{\infty}\right)$; this by restricting to Seifert surfaces, this implies that $(V, \varphi)$ is a boundary over $\mathbf{Z}_{d}$ for $V$ a Seifert surface of $K$. Hence, for any $d, \alpha(K, \varphi)$ is trivial in $\mathbf{Q} R / R$. For $d$ a prime power, we use one of the principal results of $[\mathbf{R 1}]$, namely that $\alpha(K, \varphi)$ may be computed as follows in terms of the cohomology of $A_{\infty}$ : The induced $\mathbf{Z}_{d}$ cover of $A_{\infty}, \widetilde{A_{\infty}}$, satisfies $2 n$ dimensional Poincaré duality as if it were a manifold with boundary. In particular, $H_{2 n}\left(\widetilde{A_{\infty}}, \partial \widetilde{A_{\infty}} ; \mathbf{C}\right)=\mathbf{C}$, and so the cup product defines a Hermitian form on $H^{n}\left(\widetilde{A_{\infty}}, \partial \widetilde{A_{\infty}} ; \mathbf{C}\right)$. The signatures $\sigma_{j}$ of the restriction of this form to the $\omega^{j}$ eigenspace of the action of $\mathbf{Z}_{d}$ on the cohomology then give the alpha-invariant of $K$ via the formula

$$
\alpha(K, \varphi)=\sum_{i=1}^{d-1} \sigma_{j} \chi^{-j} .
$$

But $A_{\infty}$, and hence $\widetilde{A_{\infty}}$, is a product, because it is a covering of the exterior of a disk knot which is a product. It is easy to see from this that all cup products must vanish, and therefore that all the signatures $\sigma_{j}$ are zero.

There are a variety of knots to which Theorem 4.9 applies.

EXAMPLE 4.10. For $n$ greater than two and $p$ a prime, let $K$ be a doubled disk knot with group $\pi$ given by an extension of $\mathbf{Z}$ by $\mathbf{Z}_{p}$, as provided by Proposition 3.1. The surgery group $L_{2 n+1}(\pi)$ is the kernel of $1-t_{*}: L_{2 n}\left(\mathbf{Z}_{p}\right) \rightarrow L_{2 n}\left(\mathbf{Z}_{p}\right)$. Let $A$ be an element of $L_{2 n+1}(\pi)$ whose image in $L_{2 n}\left(\mathbf{Z}_{p}\right)$ has nontrivial multisignature $\rho(A)$. For $n$ even, and the monodromy of the extension given by multiplication by $-1, t_{*}$ is the identity, so there are many such elements $A$. For $n$ odd, we choose $p=7$, and proceed as in Example 1.6 to find an explicit element in the kernel. Realizing $A$ by a cobordism of $X$ produces a new knot $K^{\prime}$ with exterior $X^{\prime}$, whose $\alpha$-invariant differs from that of $K$ by $\rho(A)$. (This follows from the definition of the map $L_{2 n+1}(\pi) \rightarrow L_{2 n}\left(\mathbf{Z}_{p}\right)$ which comes from codimension-one splitting.) It is then straightforward to use Theorem 4.9 to show that $K^{\prime}$ is not a doubled disk knot.

For $n=2$, this procedure will not work, as a knot in $S^{4}$ which has a group such as $\pi$ will not even be algebraically doubly slice, let alone a doubled disk knot. Instead, Let $L$ and $L^{\prime}$ be homotopy equivalent lens spaces which are not diffeomorphic but have the same $\mu$-invariant. These correspond to knots $K$ and $K^{\prime}$ in $S^{4}$. The knot $K \#-K^{\prime}$ has exterior homotopy equivalent to that of the doubled disk knot $K \#-K$, and so is doubly slice. Then Theorem 4.9 can be used to show that $K \#-K^{\prime}$ is not a doubled disk knot.

REMARK. Another way of finding obstructions to knots being doubled disk knots is based on the observation that such knots have a symmetry given by reflection in the sphere along which the disks are doubled. Since this reflection is 
orientation reversing, one sees that a doubled disk knot is (-) amphicheiral. The last section of the author's paper [R1] shows how the even-dimensional CassonGordon invariants give obstructions to amphicheirality, and thereby to being a doubled disk knot. This amphicheirality condition is not as strong as the one given in Theorem 4.9, however. Consider, for instance a fibred knot in $S^{4 k+1}$ with fiber the connected sum of two homotopy equivalent lens spaces. It is not hard to show that a $4 k-1$ dimensional lens space admits an orientation reversing homeomorphism, so that such a knot will be $(-)$ amphicheiral. (Using [W], this is true even for nonlinear lens spaces of this dimension.) However, one can combine the 'hyperbolicity' condition of $[\mathbf{L}]$ with Theorem 4.9 to show that if the $\alpha$-invariants of the lens spaces are different, the resulting knot will not be the double of a disk knot.

\section{REFERENCES}

[AM] S. Akbulut and J. McCarthy, Lectures on Casson's invariant for homology spheres, Preprint, Michigan State University.

[AS] M. Atiyah and I. Singer, The index of elliptic operators: III, Ann. of Math. (2) 87 (1968), 546-604.

[B] W. Browder, Surgery on simply-connected manifolds, Springer-Verlag, Berlin and New York, 1970.

[CR] S. Cappell and D. Ruberman, Imbeddings and homology cobordisms of lens spaces, Comment. Math. Helv. (to appear).

[CS1] S. Cappell and J. Shaneson, Homology surgery and the codimension-two placement problem, Ann. of Math. (2) 99 (1974), 277-348.

[CS2] _ _ Pseudo-free actions. I, Lecture Notes in Math., vol. 763, Springer, Berlin and New York, 1979, pp. 395-447.

[C] A. Casson, An integer invariant of homology 3-spheres (to appear).

[CG1] A. Casson and C. Gordon, Cobordism of classical knots, preprint, Orsay, 1975.

[CG2] _, On slice knots in dimension three, Proc. Sympos. Pure Math., vol. 32, Amer. Math. Soc., Providence, R.I., 1978.

[GL] P. Gilmer and C. Livingston, On embedding 3-manifolds in 4-space, Topology 22 (1983), 241252.

[HW] G. H. Hardy and E. M. Wright, An introduction to the theory of numbers, Oxford Univ. Press, Oxford, 1938.

[K] M. Kervaire, Les noeuds des dimensions superieures, Bull. Soc. Math. France 93 (1965), 225271.

[L] J. Levine, Doubly sliced knots and doubled disk knots, Michigan Math. J. 30 (1983), 249-256.

[M] J. Milnor, Infinite cyclic coverings, Topology of Manifolds (J. Hocking, ed.), Prindle, Weber \& Schmidt, Boston, Mass., 1968.

[P] P. Petrie, The Atiyah-Singer invariant, the Wall groups $L_{n}(\pi, 1)$, and the function $\left(\right.$ te $^{x}+$ 1) / $\left(t e^{x}-1\right)$, Ann. of Math. 92 (1970), 174-187.

[R1] D. Ruberman, Doubly slice knots and the Casson-Gordon invariants, Trans. Amer. Math. Soc. 279 (1983), 569-588.

[R2] _ Imbedding punctured lens spaces and connected sums, Pacific J. Math. 113 (1984), 481491.

[S1] L. Smolinsky, Doubly slice knots which are not the double of a disk knot, Trans. Amer. Math. Soc. 298 (1986), 723-732.

[S2] _ Casson-Gordon invariants of some 3-fold branched covers of knots.

[St] N. Stoltzfus, Isometries of inner product spaces and their geometric applications, Geometric Topology (J. C. Cantrell, ed.), Academic Press, New York, 1979.

[Su] D. Sumners, Invertible knot cobordisms, Comment. Math. Helv. 46 (1971), 240-256.

[W] C. T. C. Wall, Surgery on compact manifolds, Academic Press, New York and London, 1970.

Department of Mathematics, Brandeis University, Waltham, MassachuSETTS 02254 